\title{
Heterogeneity in SARS-CoV-2 Positivity by Ethnicity in Los Angeles
}

\author{
Lao-Tzu Allan-Blitz ${ }^{1}$ (D) - Fred Hertlein ${ }^{2} \cdot$ Jeffrey D. Klausner ${ }^{3}$ \\ Received: 22 February 2021 / Revised: 14 May 2021 / Accepted: 17 May 2021 / Published online: 24 May 2021 \\ (C) W. Montague Cobb-NMA Health Institute 2021
}

\begin{abstract}
Recent studies have identified notable disparities in SARS-CoV-2 infection risk among ethnic minorities. We evaluated SARSCoV-2 test results from individuals presenting for testing in Los Angeles between June and December, 2020. We calculated prevalence ratios for various employment categories. Among 518,914 test results, of which 295,295 (56.9\%) were from individuals reporting Hispanic ethnicity, SARS-CoV-2 positivity was $16.5 \%$ among Hispanic individuals compared to $5.0 \%$ among non-Hispanic individuals $(p$-value $<0.01)$. The prevalence ratios comparing Hispanic and non-Hispanic individuals were highest for members of the media ( $\mathrm{PR}=6.7$; 95\% CI 4.3-10.4), government employees ( $\mathrm{PR}=4.0$; 95\% CI 3.3-4.9), and agricultural workers ( $\mathrm{PR}=4.0 ; 95 \%$ CI 3.2-5.0). Such heterogeneity warrants further investigation in order to develop targeted public health interventions towards specific drivers of SARS-CoV-2 transmission.
\end{abstract}

Keywords SARS-CoV-2 $\cdot$ Healthcare disparities $\cdot$ California

\section{Background}

The impact of the SARS-CoV-2 pandemic has been disproportionately felt among Hispanic communities [1-3]. The complex and interconnected structural and systemic factors that likely contribute to such disparities [4] are also likely driving the heterogeneity with which SARS-CoV-2 spreads [5, 6]. Ironically, however, the broad business closures enforced in order to curb the pandemic may be less efficacious among minority and poorer communities, as such communities are less able to limit their mobility (e.g., working from home or limit within household contact due to dense housing and household occupancy) and are less likely to work in settings with regular testing or adequate ventilation, all of which may increase risk of SARS-CoV-2 infection [7]. Even worse, such communities who have less financial security at the outset may feel the economic impacts of public health interventions most acutely $[8,9]$.

Lao-Tzu Allan-Blitz

lallan-blitz@partners.org

Jeffrey D. Klausner

jdklausner@med.usc.edu

1 Department of Medicine, Brigham and Women's Hospital, 75 Francis Street, Boston, MA 02115, USA

2 Curative Inc., San Dimas, CA, USA

3 Department of Preventive Medicine, Keck School of Medicine, University of Southern California, Los Angeles, CA 90033, USA
A new strategy, therefore, is desperately needed. In the wake of previous epidemics, targeted public health interventions have been made possible via a thorough understanding of factors that facilitate the transmission of disease. In response to the human immunodeficiency virus (HIV) epidemic, for example, the HIV Prevention Trials Network has developed innumerable, novel, and cost-effective interventions that are tailored directly towards factors that drive infection [10]. Similarly, targeted interventions may be possible for the SARS-CoV-2 pandemic if we are able to develop a more thorough understanding of the specific drives among subpopulations at highest risk.

We therefore aimed to evaluate the risk of SARSCoV-2 infection associated with different employment categories among Hispanic compared to White individuals, in order to unwind the details of factors that contribute to the disparity in burden of infection. We performed that analysis among a community-based sample in Los Angles as Los Angeles county is among the most populous counties in the USA and one of the most diverse, with $48.6 \%$ of those surveyed in the national census identifying as Hispanic [11].

\section{Methods}

We collected data from individuals presenting for SARS-CoV-2 polymerase chain reaction testing to one of 209 ad hoc drive- 
through centers across 68 zip codes in Los Angeles county. Individuals presented between June and December, 2020. Those dates were selected for practical reasons as that was when testing data made available to us. Specimens were collected via oral swabs and processed as has been previously reported [12]. Data collected at the time of testing as a part of routine practice included age, gender, race and ethnicity, reported exposure to an individual known to be infected with SARS-CoV-2, report of symptoms (muscle aches, cough, fevers, diarrhea, nausea, headache, congestion, sore throat, anosmia, and shortness of breath), and type of employment from a pre-specified list. Laboratory data collected included human and viral cycle threshold values, date, and time of testing.

We excluded from our analysis individual tests for which there were missing values for ethnicity $(n=165,994)$, employment category ( $\mathrm{n}=1,188,036)$, or indeterminate test results ( $\mathrm{n}$ $=4728$ ). We then calculated the prevalence of SARS-CoV-2 test positivity by ethnicity and employment category as well as prevalence ratios and $95 \%$ confidence intervals (CI) for each employment category. We specified our analysis specifically among those of Hispanic heritage given the notable disparities reported among that population [3, 13], and the notable proportion of individuals of Hispanic heritage in Los Angeles [11]. The Mass General Brigham institutional review board deemed the analysis of de-identified data did not constitute human subjects' research (2020P003530).

\section{Results}

Overall, we analyzed 518,914 individual test results, of which 296,052 (57.1\%) were collected from women and 295,295 (56.9\%) reported Hispanic ethnicity. The mean age of the population was 35 years (standard deviation 15.2). In all, $247,974(47.8 \%)$ individuals reported any symptoms at the time of testing. SARS-CoV-2 positivity was $16.5 \%$ among Hispanic individuals compared to $5.0 \%$ among non-Hispanic individuals $(p$-value $<0.01)$. The percent of individuals who reported symptoms at the time of testing among those who tested positive was comparable among Hispanic (70.9\%) and nonHispanic individuals $(69.8 \%)$.

Table 1 shows the SARS-CoV-2 positivity by employment category as well as prevalence ratios and 95\% confidence intervals. All prevalence ratios were $>1$ and statistically significant. Most notably, agriculture workers (PR 4.0; 95\% CI 3.2-5.0), government
Table 1 SARS-CoV-2 positivity among Hispanic and nonHispanic individuals, Los Angeles, June-December, 2020

\begin{tabular}{llll}
\hline & \multicolumn{2}{l}{ SARS-CoV-2 positivity } & \\
\cline { 2 - 4 } & $\begin{array}{l}\text { Hispanic } \\
(\mathrm{n}=295,295)\end{array}$ & $\begin{array}{l}\text { Non-Hispanic } \\
(\mathrm{n}=223,619)\end{array}$ & Prevalence ratio (95\% CI) \\
\hline Overall Employment* $(\mathrm{n}=518,914)$ & $16.5 \%$ & $5.0 \%$ & $3.3(3.2-3.4)$ \\
Agriculture $(\mathrm{n}=4415)$ & $25.9 \%$ & $6.5 \%$ & $4.0(3.2-5.0)$ \\
Construction $(\mathrm{n}=23,584)$ & $16.4 \%$ & $5.0 \%$ & $3.2(2.9-3.6)$ \\
Correctional facility $(\mathrm{n}=2038)$ & $18.3 \%$ & $7.1 \%$ & $2.6(1.9-3.4)$ \\
Delivery or ride-share $(\mathrm{n}=16,746)$ & $12.4 \%$ & $3.2 \%$ & $3.9(3.3-4.3)$ \\
Disability care $(\mathrm{n}=18,829)$ & $18.1 \%$ & $8.7 \%$ & $2.1(1.9-2.3)$ \\
Education $(\mathrm{n}=59,595)$ & $13.1 \%$ & $3.6 \%$ & $3.6(3.4-3.9)$ \\
Elderly care $(\mathrm{n}=10,294)$ & $9.0 \%$ & $2.7 \%$ & $3.3(2.8-4.1)$ \\
Emergency services $(\mathrm{n}=13,173)$ & $14.4 \%$ & $5.7 \%$ & $2.5(2.2-2.9)$ \\
Frist responder $(\mathrm{n}=15,150)$ & $11.6 \%$ & $4.2 \%$ & $2.8(2.4-3.1)$ \\
Food services $(\mathrm{n}=85,497)$ & $21.2 \%$ & $6.5 \%$ & $3.3(3.1-3.4)$ \\
Government personnel $\dagger(\mathrm{n}=13,646)$ & $7.3 \%$ & $1.8 \%$ & $4.0(3.3-4.9)$ \\
Grocery store $(\mathrm{n}=30,818)$ & $12.7 \%$ & $3.5 \%$ & $3.6(3.2-4.0)$ \\
Health care $(\mathrm{n}=157,684)$ & $14.0 \%$ & $4.7 \%$ & $3.0(2.9-3.1)$ \\
Member of media $(\mathrm{n}=4715)$ & $5.3 \%$ & $0.8 \%$ & $6.7(4.3-10.4)$ \\
Public transportation $(\mathrm{n}=9039)$ & $20.1 \%$ & $9.1 \%$ & $2.2(1.9-2.5)$ \\
Retail or manufacturing $(\mathrm{n}=92,958)$ & $20.8 \%$ & $6.9 \%$ & $3.0(2.9-3.1)$ \\
\hline
\end{tabular}

The table shows the SARS-CoV-2 test positivity among Hispanic and non-Hispanic individuals by type of employment as well as the prevalence ratio and $95 \%$ confidence intervals for each employment category

*Data were collected beginning September 2020

$\dagger$ Self-defined and inclusive of any employment in the local, state, or federal government 
personnel (PR 4.0; 95\% CI 3.3-4.9), and members of the media (PR 6.7; 95\% 4.3-10.4) had increased SARS-CoV-2 prevalence ratios among Hispanic compared to non-Hispanic individuals.

\section{Discussion}

From a community-based sample of individuals presenting for testing in Los Angeles county, we identified a significant disparity in SARS-CoV-2 test positivity among Hispanic compared to non-Hispanic individuals. And though descriptive, we also identified notable heterogeneity in risk based on reported employment among Hispanic communities.

Many recent studies have demonstrated the disproportionate burden of SARS-CoV-2 infection suffered by minority races and ethnicities [1-4, 14-17]. There are numerous factors that likely contribute to those findings, including the higher proportion of immigrant families without health insurance limiting the ability to seek care, and language barriers impacting the quality of care received, all of which may increase the time to diagnosis and subsequently increase opportunities for transmission. Financial insecurity may also result in the need for potentially higher-risk employment or limiting the ability to work from home [18]. Housing insecurity leading to denser household occupancy [19] also increases the risk for transmission [20]. Furthermore, the impact of unemployment on the risk for SARS-CoV-2 infection is unclear; however, the rates of unemployment were higher among Hispanic compared to White individuals during the study period [21]. That may be a valuable subject for future research.

The tangled web of those factors as well as innumerable others creates significant challenges in developing public health strategies that optimally address the needs of such communities. In order to untangle such a web, we must first be able to precisely describe the specific scenarios that perpetuate those disparities. Testing centers can be used to gather detailed exposure data (e.g., exposure to gyms, places of worship, parks, museums, hospitals, nursing homes, overnight camps, hotels, beaches, movie theaters, bars, restaurants, and airports) as well as dates of those exposures in order to understand when and how the transmission chains occur. Furthermore, such data should be collected among individuals who test positive as well as negative, as the comparison of risk will be essential in evaluating which types of exposures truly drive transmission.

Our results demonstrated notably higher prevalence ratios for Hispanic individuals employed as members of the media, agriculture workers, and government personnel compared to non-Hispanics with similar employment. A recent study similarly found increased risk for SARS-CoV-2 positivity among kitchen workers of minority races [22]. There are likely associations between socioeconomic status and the reported employment categories, which contribute to the observed effect. Unfortunately, our data preclude controlling for household income, but we hypothesize that non-Hispanic individuals within the same job might be of higher socioeconomic status, and thus less exposed to SARS-CoV-2 due to greater ability to work from home and live in less crowded households.

Beyond the known socioeconomic and structural inequities that most likely contribute to such differences, there may be ongoing disparities in division of labor within the workforce of essential workers in specific roles that additionally compounds the heighted risk for transmission. For example, Hispanic members of the media may be more likely than non-Hispanic members to work in settings that place them at higher risk for SARS-CoV-2 transmission and acquisition. One survey found that Hispanic individuals were more likely than White individuals to lose their job during the pandemic [23], which may suggest that the jobs held by Hispanic individuals were different in substance than their White counterparts, and thus reflect ongoing discrepant positions held by different ethnicities. We did not have sufficient data, however, to evaluate other confounding factors or the division of labor within the workforce by ethnic heritage; thus, further research is needed to more deeply evaluate such findings.

Our study was also limited in that all participants were tested in one geographic region, thus potentially limiting the generalizability of our results. However, given the diversity within Los Angeles (mirrored partially among our sample), and the fact that Los Angeles in particular is an important case study of the ongoing socioeconomic disparities surrounding the SARS-CoV-2 pandemic, we do not feel that limitation reduces the importance of our results. Our study is additionally limited in that we were unable to adjust our analysis based on socioeconomic status, and thus our results may be confounded; thus, further research is warranted.

\section{Conclusion}

Our results add further support to the existing body of research identifying notable disparities in SARS-CoV-2 test positivity among ethnic minorities. Furthermore, in an attempt to explore the heterogeneity in SARS-CoV-2 transmission within Hispanic compared to non-Hispanic communities, we reported prevalence ratios for various employment categories, noting that several demonstrated significantly higher risk for infection. Further research is needed in order to precisely identify factors driving transmission within high-risk populations and thereby develop tailored public health interventions.

Acknowledgements The authors would like to acknowledge the city of Los Angeles. 


\section{Declarations}

Ethics Approval and Consent to Participate This article does not contain any studies with human participants performed by any of the authors. The Mass General Brigham institutional review board deemed the analysis of de-identified data did not constitute human subjects' research (2020P003530). No informed consent was necessary.

Conflict of Interest Dr. Allan-Blitz has served as a consultant for Curative Inc. Dr. Klausner has served as the medical director of Curative Inc. during the observation period. Fred Hertlein has served as an employee of Curative Inc. during the observation period.

\section{References}

1. Ogedegbe G, Ravenell J, Adhikari S, Butler M, Cook T, Francois F, et al. Assessment of racial/ethnic disparities in hospitalization and mortality in patients with COVID-19 in New York City. JAMA Netw Open. 2020;3(12):e2026881.

2. Anand S, Montez-Rath M, Han J, Bozeman J, Kerschmann R, Beyer P, et al. Prevalence of SARS-CoV-2 antibodies in a large nationwide sample of patients on dialysis in the USA: a crosssectional study. Lancet. 2020;396:1335-44.

3. Macias Gil R, Marcelin JR, Zuniga-Blanco B, Marquez C, Mathew T, Piggott DA. COVID-19 pandemic: disparate health impact on the Hispanic/Latinx population in the United States. J Infect Dis. 2020;222:1592-5.

4. Page KR, Flores-Miller A. Lessons we've learned-Covid-19 and the undocumented Latinx community. N Engl J Med. 2021;384(1):5-7.

5. Britton T, Ball F, Trapman P. A mathematical model reveals the influence of population heterogeneity on herd immunity to SARSCoV-2. Science. 2020;369(6505):846-9.

6. Centers for Disease Control and Prevention. COVID data tracker: integrated county view. Available at: https://covid.cdc.gov/coviddata-tracker/?CDC_AA_refVal=https $\% 3 \mathrm{~A} \% 2 \mathrm{~F} \% 2 \mathrm{Fwww} . \mathrm{cdc}$. gov $\% 2 \mathrm{~F}$ coronavirus $\% 2 \mathrm{~F} 2019$-ncov $\% 2 \mathrm{Fcases}$-updates $\% 2 \mathrm{~F}$ casesin-us.html\#county-view Accessed 11/30/20. 2020

7. Chang SPE, Wei Koh P, Geradin J, Redbird B, Grusky D, Leskovec J. Mobility network models of COVID-19 explain inequities and inform reopening. Nature. 2020. https://doi.org/10.1038/ s41586-020-2923-3.

8. Usman M, Ali Y, Riaz A, Riaz A, Zubair A. Economic perspective of coronavirus (COVID-19). J Public Aff. 2020:e2252

9. Nicola M, Alsafi Z, Sohrabi C, Kerwan A, al-Jabir A, Iosifidis C, et al. The socio-economic implications of the coronavirus pandemic (COVID-19): a review. Int J Surg. 2020;78:185-93.

10. HIV Prevention Trials Network. Available at: https://www.hptn. org/ Accessed February 3, 2021

11. United States Census Bureau. Table B03002. Hispanic or Latino Origin by Race. Available at: https://data.census.gov/cedsci/table?
$\mathrm{q}=\mathrm{B} 03002 \& \mathrm{~g}=0500000 \mathrm{US} 06037 \& \mathrm{tid}=\mathrm{ACSDT} 1 \mathrm{Y} 2019$. B03002\&hidePreview=true Accessed February 2, 2021

12. Kojima N, Turner F, Slepnev V, et al. Self-collected oral fluid and nasal swab specimens demonstrate comparable sensitivity to clinician-collected nasopharyngeal swab specimens for the detection of SARS-CoV-2. Clin Infect Dis. 2020. https://doi.org/10. 1093/cid/ciaa1589.

13. Bruckner TA, Parker DM, Bartell SM, Vieira VM, Khan S, Noymer A, et al. Estimated seroprevalence of SARS-CoV-2 antibodies among adults in Orange County, California. Sci Rep. 2021;11(1):3081.

14. Wadhera RK, Wadhera P, Gaba P, Figueroa JF, Joynt Maddox KE, Yeh RW, et al. Variation in COVID-19 hospitalizations and deaths across New York City boroughs. JAMA. 2020;323(21):2192-5.

15. Killerby MEL-GR, Haight SC, Schrodt CA, England L, Gomes DJ, Shamout M, et al. Characteristics associated with hospitalization among patients with COVID-19-Metropolitan Atlanta, Georgia, March-April 2020. MMWR Morb Mortal Wkly Rep. 2020;69: 790-4.

16. Price-Haywood EG, Burton J, Fort D, Seoane L. Hospitalization and mortality among Black patients and White patients with Covid19. N Engl J Med. 2020;382(26):2534-43.

17. Millett GA, Jones AT, Benkeser D, Baral S, Mercer L, Beyrer C, et al. Assessing differential impacts of COVID-19 on black communities. Ann Epidemiol. 2020;47:37-44.

18. News Release: Bureau of Labor Statistics. US Department of Labor. Job Flexibilities and Work Schedules 2017-2018. Published September 2019. Available at: https://www.bls.gov/ news.release/pdf/flex2.pdf Accessed February 5th, 2021

19. Burr JA, Mutchler JE, Gerst K. Patterns of residential crowding among Hispanics in later life: immigration, assimilation, and housing market factors. J Gerontol B Psychol Sci Soc Sci. 2010;65(6): 772-82.

20. Cerami C, Rapp T, Lin FC, et al. High household transmission of SARS-CoV-2 in the United States: living density, viral load, and disproportionate impact on communities of color. medRxiv. 2021.

21. Economic Research: FRED Graph. Unemployment rate White and Hispanic or Latino. Data from U.S. Bureau of Labor Statistics. Available at: https://fred.stlouisfed.org/series/CALOSA7URN\#0 Accessed May 14th 2021

22. Chen YH GM, Riley A, Balmes J, Duchowny K, Harrison R, Matthay E, Bibbins-Domingo K. Excess mortality associated with the COVID-19 pandemic among Californians 18-65 years of age, by occupational sector and occupation: March through October 2020. medRxiv. Available at: https://www.medrxiv.org/content/ 10.1101/2021.01.21.21250266v1.full.pdf. 2021

23. Guskin E. Hispanics are almost twice as likely as Whites to have lost their jobs amid the pandemic, poll finds. The Washington Post. 2020

Publisher's Note Springer Nature remains neutral with regard to jurisdictional claims in published maps and institutional affiliations. 